\title{
Safety and efficacy of subcutaneous adrenaline as a treatment for anaphylactic reactions to polyvalent antivenom
}

Antivenom serum (AVS) is widely accepted as an effective form of treatment in snake envenoming [1]. However, anaphylactic and pyrogenic reactions are common for both polyvalent and monovalent AVS [14]. Many premedications including antihistamines and hydrocortisone have been used to minimise such reactions without much benefit $[2,3]$. Premedication with low dose adrenaline subcutaneously (sc) has shown a significant reduction of the acute adverse reactions to AVS [4]. A major concern with the use of adrenaline pre-treatment is the risk of intracerebral haemorrhage and the potential danger in children, pregnancy and in heart disease $[5,6]$. Published data on the safety of adrenaline in the treatment of established AVS reactions are sparse. Accordingly, we planned this study to assess the safety and efficacy of adrenaline as the sole treatment of moderate to severe AVS reactions. The sc route was used because it was considered safer than the intramuscular (im) route.

This study was conducted at the General Hospital, Anuradhapura, in the North Central Province of Sri Lanka from April to May 2002. The subjects were 36 patients who had developed reactions to the polyvalent antivenom (lyophilised enzyme refined, manufactured in India, Batch no: AVS30-2001).

The reactions were catergorised as mild, moderate and severe based on the criteria of a former study [3]. Twenty-one patients with moderate to severe reactions qualified to receive adrenaline. The AVS reaction profile, the blood pressure and the pulse rate in particular, was recorded just before the treatment and observed for $2 \mathrm{~h}$ initially and 4 hourly for $48 \mathrm{~h}$. These reactions were treated initially by discontinuing the AVS infusion temporarily and administering $0.5 \mathrm{~mL}(1: 1000)$ of adrenaline subcutaneously. Data such as age and sex, previous history of eczema, catarrh, urticaria, allergy, nature of the snake bite and other medical conditions were also recorded.
Ethical approval was obtained from the Research and Ethical Committee of the Faculty of Medicine, University of Peradeniya.

Mean values of the pre-treatment and post-treatment blood pressure, pulse rate and time of blood pressure rise were calculated. The mean difference between pre- and post-treatment systolic BP, diastolic BP, and pulse rate were compared by using paired t-test. Data analysis was done by using the Windows-based SPSS statistical package, standard version 10.0.1.

The severity of the AVS reactions were: mild $15(28.8 \%)$, moderate $19(36.5 \%)$ and severe $2(3.9 \%)$. The 21 cases (17 males) with moderate to severe reactions who qualified to receive adrenaline had a mean age of 34.2 years (range 18-55). The mean blood pressure values and mean pulse rates of pre-treatment and post-treatment were tabulated (Table 1). The differences between the pre- and post-systolic BP, diastolic BP and pulse rate were statistically significant with $\mathrm{p}$ values $<0.001$ (Table 2 ).

Table 1. Blood pressure response with adrenaline ( $n=21$ patients)

\begin{tabular}{lccccc}
\hline & Minimum & Maximum & Mean & SD & SEM \\
\hline Pre-SBP (mmHg) & 0 & 130 & 80 & 27.9 & 6.0 \\
Pre-DBP (mmHg) & 0 & 90 & 46.6 & 26.7 & 5.8 \\
Post-SBP (mmHg) & 90 & 150 & 114.7 & 13.6 & 3.0 \\
Post-DBP (mmHg) & 50 & 90 & 73.9 & 9.2 & 2 \\
Pre-Pulse (/min) & 72 & 140 & 107 & 16.9 & 3.7 \\
Post-Pulse (/min) & 60 & 120 & 85.8 & 17.3 & 3.8 \\
\hline
\end{tabular}

SBP-systolic blood pressure, DBP-diastolic blood pressure, Pulse-radial pulse rate 
The mean time of rise in blood pressure was $10.24 \mathrm{~min}$ with $95 \%$ confidence interval of 6.63 to 13.85 . The other reactions have taken a variable duration of time to recover, itching and urticaria taking longest duration. All the patients recovered uneventfully.

The management of AVS reactions has become a part and parcel of the management of snake envenoming. Adrenaline, an $\boldsymbol{\alpha}$ - and $\boldsymbol{\beta}$-adrenoceptor stimulator, given $\mathrm{im}$ is the drug of choice for anaphylaxis in general, because of its cardiovascular and bronchial effects and the speed of action. It may also stabilise mast cell membranes and reduce the release of vasoactive autacoids [6]. These pharmacological effects are generally beneficial, but could be harmful occasionally if absorption of the drug occurs fast particularly by im route. In this study, we used the sc route instead of the im route.

Table 2. Paired analysis of pre- and post-treatment parameters with adrenaline

\begin{tabular}{lccc}
\hline Paired parameter & t-value & P-value & $\begin{array}{c}95 \% \text { confidence } \\
\text { interval }\end{array}$ \\
\hline Pair 1 (SBP) & 6.172 & $<0.0001$ & 46.51 to 23.01 \\
Pair 2 (DBP) & 4.527 & $<0.0001$ & 39.79 to 14.69 \\
Pair 3 (Pulse) & 4.654 & $<0.0001$ & 11.72 to 30.76 \\
\hline
\end{tabular}

SBP, DBP as in Table 1

Our study provides data to show that adrenaline sc is an effective and safe method of treating AVS reactions. It normalised the blood pressure and pulse rate in moderate to severe AVS reactions without causing a surge of blood pressure or tachycardia. Despite observed immediate blood pressure rise, normalisation of blood pressure occurred gradually (mean $10.2 \mathrm{~mm}$ ), which could be considered beneficial as a sudden surge may lead to intracerebral haemorrhage.

Subcutaneous adrenaline is effective in normalising blood pressure and pulse rate in moderate to severe reactions to antivenom serum without causing adverse reactions such as surges of blood pressure or tachycardia.

\section{References}

1. Ariaratnam CA, Sjostrom L, Raziek Z, Kularatne SAM, Kodikaraarachchi RWK, et al. An open, randomized comparativetrial of two antivenoms for the treatment of envenoming by Sri Lankan Russell'sviper (Daboia Russelli Russelli). Transactions of the Royal Society of Tropical Medicine and Hygiene 2000; 95: 74-80.

2. Kularatne SAM. Reaction to snake venom antisera: study of pattern, severity, and management at General Hospital, Anuradhapura. Sri Lanka Journal of Medicine 2000; 9: 8-13.

3. Seneviratne SL, Opanayaka CJ, Ratnayake NS, Sarath Kumara KE, Sugathadasa AM, et al. Use of antivenom serum in snake bite: a prospective study of hospital practice in the Gampaha district. Ceylon Medical Journal 2000; 45: $65-8$.

4. Premawardhena AP, de Silva CE, Fonseka MM, Gunatilake $\mathrm{SB}$, de Silva HJ. Low dose subcutaneous adrenaline to prevent acute adverse reactions to antivenom serum in people bitten by snakes: randomized, placebo controlled trial. British Medical Journal 1999; 318: 1041-3.

5. Dassanayake AS, Karunanayake P, Kasturiratne KTAA, Fonseka MMD, Wijesiriwardena B, et al. Safety of low dose subcutaneous adrenaline used for prophylaxis against acute adverse reactions to anti-venom serum (AVS) in patients bitten by snakes. Ceylon Medical Journal 2002; 47: 48-9.

6. Laurence DR, Bennet PN, Brown MJ, eds. Clinical Pharmacology. London: Churchill Livingstone, 1977: 412-24.

SAM Kularatne, IB Gawarammana, PVR Kumarasiri, N Senanayake, WP Dissanayake and H Ariyasena, Departments of Medicine and Community Medicine, Faculty of Medicine Peradeniya, and General Hospital, Anuradhapura, Sri Lanka Correspondence: SAMK, Department of Medicine, Faculty of Medicine, University of Peradeniya, Peradeniya, Sri Lanka. Tel: 00948 388368, Fax: 00948 232343, e-mail: samkul@sltnet.lk. (Competing interests: none declared). Received 17 March and revised version accepted 14 September 2003. 